Review

\title{
The Role of Non-Steroidal Anti-Inflammatory Drugs in the Chemoprevention of Breast Cancer
}

\section{Sarah L. Horn * and Ian S. Fentiman}

Hedley Atkins Breast Unit, Guy’s Hospital, London SE1 9RT, UK; E-Mail: Ian.Fentiman@gstt.nhs.uk

* Author to whom correspondence should be addressed; E-Mail: raheresarah@btinternet.com; Tel.: +44-207-188-7188; Fax: +44-207-403-8381.

Received: 31 March 2010; in revised form: 26 April 2010 / Accepted: 10 May 2010 /

Published: 17 May 2010

\begin{abstract}
Epidemiological evidence suggests that non-steroidal anti-inflammatory drugs (NSAIDs) which act as cyclooxygenase (COX-2) inhibitors may reduce breast cancer incidence by up to $20 \%$. These agents are often taken for pain relief by older women with osteoarthritis. Age is the major risk factor for breast cancer in women with $50 \%$ cases being diagnosed in those aged $>65$ years. NSAIDs reduce serum estradiol by $17 \%$ in post-menopausal women and since most of these who develop breast cancers have estrogen receptor positive tumours; this suggests a possible preventative role. Careful use of these agents could provide a strategy for both relief of symptoms of osteoarthritis and also breast cancer prevention. Instead of conducting a randomised trial, proof of efficacy could be from an adequately powered cohort study within the breast screening programme.
\end{abstract}

Keywords: non-steroidal anti-inflammatory drugs; breast cancer; chemoprevention

\section{Introduction}

Chemoprevention is the use of a drug or intervention to interfere with pathogenesis and thereby prevent disease. In the last 20 years NSAIDS with COX-2 inhibition have shown potential in the chemoprevention of many forms of solid malignant neoplasms, including cancers of the colon, breast, lung and prostate.

Breast cancer is the most common malignancy in women in developed countries, with over half the cases being diagnosed in those over 65 years, and it is this older population that represents the fastest growing population group in Europe. The diagnosis can be psychologically devastating and thus any 
mechanism to reduce risk has a hugely important role to play in terms of both morbidity and mortality. The mainstay of breast cancer treatment is surgical intervention with chemotherapy, radiotherapy and endocrine manipulation, either in the neo-adjuvant and/or adjuvant setting. Drug treatment takes the form of tamoxifen directly acting on estrogen receptors (ER) in premenopausal women or the aromatase Inhibitors, letrozole and anastrazole, acting on the pathways causing inhibition of peripheral estrogen synthesis in the postmenopausal. To date, the main chemo preventative strategy has been the use of endocrine manipulation.

In 1986 Cuzick et al. [1] laid out the theoretical basis for the prevention of breast cancer using tamoxifen. Subsequently, in 1992 the Early Breast Cancer Trialists' Collaborative Group (EBCTCG) [2] published their meta-analysis of adjuvant studies which demonstrated a 39\% reduction in contralateral breast cancers in women taking tamoxifen (SD 9, 1p $<0.0001$ ). Two prevention trials, NSABP-P1 [3] and IBIS-1 [4] further confirmed tamoxifen's inhibitory effect on ER positive tumours but no effect on ER negative disease. Prichard et al. [5] concluded that the women deriving the greatest benefit from tamoxifen are premenopausal with a five year GAIL risk factor of $>1.66 \%$, postmenopausal women with a GAIL risk factor of more than $3 \%$ and postmenopausal without a uterus. The MORE study [6] in postmenopausal women with osteoporosis, who took placebo or raloxifene, reported that there was a $76 \%$ decrease in all breast tumours, specifically of ER positive phenotype (95\% confidence interval [CI], 0.13-0.44; $\mathrm{P}<001$ ). Bennett et al. [7] demonstrated elevated levels of prostaglandin in human breast cancer cells, making clear a potential role for NSAIDS in breast cancer chemoprevention.

Epidemiological studies of association between non-steroidal anti-inflammatory drugs, such as ibuprofen and aspirin, and breast cancer risk have yielded inconsistent results. NSAIDs are non prescription medications used to manage the pain of many diseases including musculoskeletal disease and osteoarthritis in the elderly, and aspirin in the prevention of cardiovascular disease. Because of comorbidity these indications for use are more common in the elderly population.

There is evidence, predominantly epidemiological but also experimental, that NSAIDS have a chemo preventative role in cardiovascular disease and in certain malignancies, with the most evidence in colorectal cancer.

\section{Evidence of NSAIDS Role in Cancer Prevention}

Evidence shows a chemo preventive effect for aspirin and other NSAIDS in colorectal cancer however the risk-benefit profiles for cancer prevention are insufficient and currently no definitive recommendations have been made. Aspirin is the most likely candidate for chemoprevention due to its known cardiovascular benefit and longer term safety and efficacy data. [8] The current literature does not agree on the dose and duration of aspirin, ibuprofen and selective COX-2 inhibitors, indicating the need for more research.

The greatest evidence base for the role of these drugs in cancer chemoprevention is colorectal cancer. Sulindac and selective COX-2 inhibitors such as celecoxib have been given to patients at high risk of colorectal cancer, for example those with Familial Adenomatous Polyposis syndrome, this disease having provided an excellent study population. It is only aspirin that provides cardiovascular benefit. There have been numerous problems with cancer chemoprevention trials using COX-2 
inhibitors. The APPROVE trial randomised patients with colonic polyps to rofecoxib or placebo, but in 2004 this trial was stopped due to significant cardiovascular side effects. The drug was then withdrawn from use worldwide. In the APC (Adenoma Prevention with Celecoxib) trial there was a 2.5 fold increase in the risk of fatal or non-fatal cardiovascular events and again after two years the trial was halted [9]. The preSAP trial [10] showed no difference in cardiovascular risk when patients were given placebo or celecoxib, although this was half the dose of celecoxib compared to that used in the APC trial.

Based upon meta-analysis of the published epidemiological evidence, use of either selective or nonselective COX -2 inhibiting agents significantly reduced the risk. Regular intake (of primarily $325 \mathrm{mg}$ aspirin or $200 \mathrm{mg}$ ibuprofen taken two or more times per week for at least 5 years) produced composite risk reductions of $43 \%$ for colorectal cancer, $24 \%$ for breast cancer, $28 \%$ for lung cancer and $27 \%$ for prostate cancer. A further recent series of case-control studies designed to compare selective and nonselective COX-2 inhibitors, daily intake of selective COX-2 inhibitors (20 mg celecoxib or $25 \mathrm{mg}$ rofecoxib) produced a $70 \%$ composite risk reduction across all four major malignancies compared to $60 \%$ for ibuprofen (non-selective) and 54\% for aspirin ( relatively selective COX-1 inhibition) [11].

\section{Cyclo-Oxygenases, Cancer and Mechanism of NSAIDS}

NSAIDS inhibit cyclooxygenase (COX) activity and subsequently reduce prostaglandin synthesis. Prostaglandins increase aromatase gene expression thereby stimulating estrogen biosynthesis in fat and muscle [12]. NSAIDS act by inhibiting of COX synthesis, which is the rate-limiting enzyme for prostaglandin biosynthesis. There are two genetic isoforms of COX. COX-1 is constitutively produced in most tissues and COX-2, the inducible form induced by pro-inflammatory stimuli [13]. Molecular studies have highlighted a role for COX-2 over expression in tumourogenesis, including breast cancer. COX-2 over-expression is a critical component in angiogenesis, mutagenesis, inhibition of apoptosis and aromatase-catalysed oestrogen biosynthesis together with immunosupression $[14,15]$ thus stimulating tumour growth [16]. High levels of COX-2 expression are evident throughout tumourogenesis in every anatomic site that has been examined [17]. Studies have observed that COX-2 over-expression is sufficient to transform normal cells to malignant neoplasms in models of carcinogenesis [18]. COX-2 is not usually detectable in normal tissues, except the testes and brain.

This process of carcinogenesis involving highly selective cellular pathways and molecular changes in response to the over-expression of COX-2 and this prostaglandin cascade is often referred to as the "inflammogenesis of cancer". COX-2 over-expression has been detected in approximately $40 \%$ of invasive breast cancer by immunohistochemistry and correlates with aggressive factors in breast cancer, including large tumour size, high histological grade, ER/PR negative and Herceptin (HER-2) over-expression $[19,20]$. COX-2 over-expression is associated with poor survival in ER/PR positive tumours but not ER/PR negative ones [21]. Use of NSAIDS has been shown to improve the survival of breast cancer patients [22]. NSAIDS, which include the non-selective aspirin and ibuprofen (COX-1 and COX-2 inhibitors) and COX-2 inhibitors (celecoxib) suppress the COX activity and reduce prostaglandin synthesis. Prostaglandins have organ-site-specific effect of increasing the levels of peripheral aromatase which stimulates oestrogen and progesterone biosynthesis [23], hence the theory behind their chemo preventative role in breast cancer. Thun et al. found evidence that NSAIDs may 
have the ability to restore apoptosis and inhibit angiogenesis, which would have an obvious therapeutic value.

Two studies in rats identified the chemoprevention of breast cancer by the selective COX-2 inhibitors, rofecoxib and celecoxib [24,25]. There is considerable evidence to suggest that COX inhibitors alone or in combination with other agents are useful in the chemoprevention of breast cancer [26]. The COX-2 inhibitor celecoxib has been shown to inhibit in vitro growth of MDA-MB-231 breast cancer cell and this cell line is estrogen receptor negative suggesting that celecoxib maybe a potential agent for the prevention of ER negative tumours. Celecoxib is a potential agent for breast cancer chemoprevention, alone or in combination with an aromatase inhibitor [27].

\section{Relationship between COX-2 and Aromatase.}

This relationship has a potential role in preventing breast cancer in older women maybe taking NSAIDS for musculoskeletal disease such as osteoarthritis and/or aspirin for cardiovascular disease prevention, that are ER positive, through inhibiting oestrogen via NSAIDS.

Experimental and epidemiological evidence suggests a significant role for the COX enzymes, particularly COX-2 in the pathogenesis of breast cancer [28]. It is known that prostaglandin E2 increases aromatase gene expression and that the synthesis of prostaglandins is catalysed by COX enzymes. Animal studies have demonstrated that aromatase activity is suppressed in COX-2 knockout mice and aromatase activity is increased by transgenic COX-2 over-expression [20,29]. COX inhibitors decrease aromatase activity in breast cancer cells and this effect starts at the transcriptional level. Real time PCR data shows that in this mechanism, promoters 1.4 and II are involved in breast cancer development. Thus prostaglandin E2 produced via COX may act locally in paracrine and autocrine fashion to increase the biosynthesis of estrogen by aromatase in hormone dependent breast cancer development [30]. There is therefore a theoretical possibility that NSAIDS will impair this sequence of COX induced PG expression and thus reduce aromatase gene expression, reducing the level of estrogen and therefore breast cancer risk. A selective COX-2 inhibitor having greater efficacy.

\section{Ongoing Clinical Trials Involving Celecoxib}

NCIC Clinical Trials Group phase III trial of examestane with or without celecoxib versus placebo in high risk postmenopausal women, are awaited with interest. REACT (Randomised European Celecoxib Trial) is a multicentre, placebo controlled, randomized phase III trial in patients with primary breast cancer. Patients, in addition to their standard adjuvant therapy, are randomized between two years celecoxib (a COX-2 inhibitor) and placebo in a 2:1 ratio in favour of celecoxib. This trial is still recruiting patients.

\section{Evidence of Breast Cancer Prevention by NSAIDS}

In 2009 a meta-analysis and systematic review was executed by Zhao et al. [31] to explore the pooled relative risk and 95\% confidence intervals of the epidemiological studies on breast cancer risk and NSAIDS. They included 26 studies with 528,705 participants. The Relative Risk (RR) of NSAID use and incidence of breast cancer was 0.94 (95\% CI: 0.88-1.00) with random effects model. A slight 
reduction of breast cancer by taking aspirin and ibuprofen was both observed with pooled RR of 0.91 (95\% CI: 0.83-0.98) and 0.81 (95\% CI; 0.67-0.97) respectively. These indicate that non-selective NSAID use is associated with a slight decrease for the development of breast cancer with a marginally statistically significant difference.

Another meta-analysis published in 2001 by Khuder et al. [32] and another in 2008 by Mangiapane et al. [33] demonstrated pooled RR of 0.82 (95\% CI: 0.75-0.89) and 0.75 (95\% CI: 0.64-0.88) respectively. There was significant heterogenecity in these studies and no clear comparison on dose and frequency of the NSAIDs. One Danish cohort study involving 28,695 women actually showed an increase risk of breast cancer compared with no use of the drugs, RR 1.27 (95\% CI: 1.10-1.45) [34]. In Zhao et al's meta-analysis from 2009 [31], sub group analysis showed the breast cancer risk reduction was higher in the case control studies (12\%) than the cohort studies (4\%) but was not statistically significant. This overview also highlighted three papers investigating the long-term use ( $>10$ years) of NSAIDS none of which showed a decrease in breast cancer development. Daily and no less than four times per week of NSAID intake were associated with risk reduction in breast cancer.

Experimental studies have also analysed the association of breast cancer risk, chemoprevention and NSAID usage. Another mechanism is the nitric oxide pathway which has anti-cancer properties by way of its anti-oxidant properties.

A study by Khalkhali-Ellis et al. [35] demonstrated that NO exerts its anticancer properties in vitro and increases production of maspin, which is inhibitory in malignant breast cancer cells. Girish et al. [36] treated 15 breast cancer patients and 15 age-matched controls with $150 \mathrm{mg}$ daily of aspirin and reported there was both an increase in serum nitric oxide and maspin levels in the breast cancer patients. This however was a very small study population making extrapolation of results very difficult.

Nitric oxide donating aspirin (NO-ASA) consisting aspirin and an -ONO2 moiety linked to it via a molecular spacer is a new drug mechanism for cancer prevention. NO-ASA inhibits a number of cancers including breast cancer and is up to 6,000x more potent than traditional aspirin, suggesting a promising agent for the future prevention and/or treatment of cancer [37]. A recent study of DNAadducts in urine demonstrates that women at high risk of, or with breast cancer, have high levels, indicating a critical role for adduct formation in breast cancer initiation. This knowledge of the first step in cancer initiation suggests it would be possible to use specific anti-oxidants that can block formation of the adducts by chemical and biochemical mechanisms. Two anti-oxidants, $\mathrm{N}$ acetylcysteine and revesterol are prime candidates to prevent breast cancer because they reduce the formation of oestrogen-DNA adducts in various in vitro and in vivo experiments [38].

\section{NSAIDS and In-Situ Disease}

COX-2 is commonly found in pre-malignant lesions, carcinoma in situ, invasive cancer and metastatic disease. High levels of expression of COX-2 are observed in premalignant lesions such as atypia of the mammary gland [39] COX-2 over-expression accounts for 63-80\% of cases in DCIS [40] The North Carolina Breast Cancer Study reported a reduced incidence of in-situ disease in women taking NSAIDs compared with controls (OR 0.7 95\% CI, 0.4-1.1) This association was weaker than invasive disease and not statistically significant. 


\section{Stage of Breast Cancer and Hormone Receptor Status}

In 2004, a case control study with 1,442 cases and 1,420 controls reported a reduction in risk of breast cancer with aspirin use among those women with hormone receptor positive tumours (OR = $0.74,95 \% \mathrm{CI}=0.60)$ but not for women with hormone receptor negative tumours $(\mathrm{OR}=0.97,95 \% \mathrm{CI}$ $=0.67-1.40)$ [12].

The California Teachers study [16] study concludes daily use of NSAIDS was not associated with breast cancer risk overall. Ibuprofen use was associated with an increased risk of breast cancer $(\mathrm{RR}=$ 1.51 , 95\% CI $=1.17-1.95$ ), and long term daily aspirin use was associated with increased risk of ER/PR negative breast cancer $(\mathrm{RR}=1.81,95 \% \mathrm{CI}=1.12-2.92)$ but it is not clear if this is causal. Among postmenopausal women, an inverse association with aspirin was seen among hormone rector positive tumours and not with hormone receptor negative tumours.

Among premenopausal women there was no statistically significant association in both hormone receptor positive and negative tumours. Other studies have highlighted that the association of NSAIDS and reduced breast cancer risk was not significantly different according to hormone receptor status [41,42]. A study by McCarthy in 2006 [43] found a significantly higher expression of COX-2 mRNA in ER and PR negative cancers, suggesting a role for COX-2 inhibitors in ER negative breast cancer patients.

A nested case control study by Sharpe et al. [44] was interpreted as showing that NSAIDs are unlikely to prevent breast cancer from developing de novo but might slow the growth of tumour, reducing the probability of detection clinically and radiologically. They highlighted protective effects of NSAIDS on incidence, tumour size and distant metastases and all associated with exposure 2-5 years before diagnosis. There was no effect on incidence of regional lymph node metastases.

\section{COX-2 Inhibitors in Combination with Aromatase Inhibitors in Metastatic Breast Cancer}

Aromatase inhibitors (AI) and COX-2 (celecoxib) inhibitors have shown promising efficacy and safety in the treatment for patients with metastatic breast cancer. Combination therapy had better or comparable efficacy compared with AI monotherapy using the end-points of progression free survival, overall response rate and, clinical benefit rate, time to progression and duration of clinical benefit, with therapies being well tolerated. There also appeared to be beneficial impact on serum lipid levels for patients receiving combination therapy in a neo adjuvant trail despite the known cardiovascular risk associated with COX-2 inhibitors. This highlights a therapeutic role of COX-2 inhibitors in breast cancer [45].

\section{Age and Breast Cancer in Older Women}

As the population as a whole now has a longer lifespan, the incidence of certain age-related cancers, such as breast cancer, will also increase. Some studies by Diadone, Gennari and another by Fischer in 1997 [46-48] have shown that older patients had tumours with more favourable biological features such as a lower proliferation rate, normal p53 and higher expression of oestrogen and progesterone receptor status and the proportion of ER positive tumours in older women being more than $80 \%$. 
Age related incidence of breast cancer in general is due to breast tissue aging rather than patient chronological age [49]. Also age itself is associated with failure of DNA repair mechanisms and is thus a major risk factor for all solid tumours. The problem however, with long term NSAID use in the elderly is the risk of NSAID related complications. These include gastrointestinal abnormalities such as peptic ulcer disease leading to GI bleeding and perforation, this can be counteracted with the concomitant use of a proton pump inhibitor.

Johnson et al. [50] performed a six year study of aspirin and other NSAID use in a cohort of 27,616 post-menopausal women with a reported risk reduction of $20 \%$ in the overall group but the benefit appearing to be with aspirin alone [(RR) 0.80, 95\% confidence interval (CI) 0.67-0.95].

A study by Sauter et al. [51] collected nipple aspirate fluid (NAF) from a small number of women (26), at increased risk of breast cancer. Both NAF and plasma samples were taken before starting celecoxib, two weeks later after 14 days of celecoxib $200 \mathrm{mg}$ twice daily and then two weeks after stopping treatment. Prostaglandin E2 levels in the NAF and serum were unchanged among premenopausal women. In contrast among the postmenopausal women at high risk there was significant reduction in PGE2 after celecoxib.

\section{Conclusions}

As has been shown, there are many potential benefits for the use of selective and non-selective NSAIDS in all aspects of breast cancer chemoprevention, along with treatment benefits in metastatic disease in combination with aromatase inhibitors. There remains a great deal of work to be done to lose the heterogenecity of the current data, especially in relation to the dose and frequency of aspirin and ibuprofen. NSAIDs have a potential significant chemoprevention role against the development of breast cancer but more clinical research is required, especially with side effect profiles of these drugs. The mortality risk and morbidity associated with these drugs would need to be carefully assessed prior to regular use in chemoprevention of breast cancer.

Epidemiological evidence suggests that non-steroidal anti-inflammatory drugs (NSAIDs) which act as COX-2 inhibitors may reduce breast cancer incidence by $20 \%$. Once again there is no consistent evidence on dose and/or frequency and duration of such drugs. NSAIDs are also used for the treatment of osteoporosis which it the most common form of arthritis occurring in $40 \%$ of women aged between 70 and 74 years. The incidence of breast cancer increases with age thus providing a potential opportunistic method of breast cancer chemoprevention with the use of NSAIDS [52]. The future still needs to be defined.

NSAIDs reduce serum estradiol by $17 \%$ in post-menopausal women. Most post-menopausal women who develop breast cancer have estrogen receptor positive tumours, suggesting a preventative role for NSAIDs. Careful use of these agents could provide a strategy for both relief of symptoms of osteoarthritis and also breast cancer prevention. Instead of conducting a randomised trial, proof of efficacy could be from an adequately powered cohort study within the breast screening programme. 


\section{Acknowledgements}

James T Evans MBBS BMedSci MRCP MRCGP.

\section{References and Notes}

1. Cuzick, J.; Wang, D.Y., Bulbrook, R.D. The prevention of breast cancer. Lancet 1986, 327, 83-86.

2. Early Breast Cancer Trialists' Collaborative Group (EBCTCG). Systemic treatment of early breast cancerby hormonal, cytotoxic, or immune therapy. Lancet 1992, 339, 71-85.

3. Fischer, B.; Constantino, J.B.; Wickherham, D.L.; Redmond, C.K.; Kavanah, M.; Cronin, W.M.; Vogel, V.; Robidoux, A.; Dimitrov, N.; Atkins, J.; Daly, M.; Wieand, S.; Tan-Chiu, E.; Ford, L.; Wolmark, N. Tamoxifen for prevention of breast cancer: Report of the National Surgical Adjuvant Breast and Bowel P-1 study. J. Natl. Cancer. Inst. 1998, 90, 1371-1388.

4. Cuzick, J.; Forbes, J.; Sestak, I.; Cawthorn, S.; Hamed, H.; Holli, K.; Howell, A. Long-term results of tamoxifen prophylaxis for breast cancer: 96 month follow-up of the randomised IBIS-1 trial. J. Natl. Cancer Inst. 2007, 99, 272-282.

5. Prichard, R.S.; Hill, A.D.; Dijkstra, B.; McDermott, E.W.; O’Higgins, N.J. The prevention of breast cancer. Br. J. Surg. 2003, 90(7), 772-783.

6. Cummings, S.R.; Eckert, S.; Krueger, K.A.; Grady, D.; Powles, T.J.; Cauley, J.A.; Norton, L.; Nickelsen, T.; Bjarnason, N.H.; Morrow, M.; Lippman, M.E.; Black, D.; Glusman, J.E.; Costa, A.; Jordan, V.C. The effect of raloxifene on risk of breast cancer in postmenopausal women: Results from the MORE randomized trial. JAMA 1999, 281, 2189-2197.

7. Bennett, A.; Berstock, D.A.; Carroll, M.A.; Stamford, I.F.; Wilson, A.J. Breast cancer, its recurrence and patient survival in relation to tumour prostaglandins. Adv. Prostaglandin Thromboxane Leukot Res. 1983, 12, 299-302.

8. Cuzick, J.; Otto, F.; Baron, J.A.; Burn, J.; Burn, J.; Greenwald, P.; Jankowski, J.; Vecchia, C.L.; Meyskens, F.; Senn, H.J.; Thun, M. Aspirin and non-steroidal anti-inflammatory drugs for cancer prevention: An international consensus statement. Lancet Oncol. 2009, 10, 501-507.

9. Pfizer Inc. 2004. Pfizer statement on new information regarding cardiovascular safety of celebrex. http://www.pfizer.com, accessed on 14 May 2010.

10. Arber, N.; Eagle, C.J.; Spicak, J.; Rácz, I.; Dite, P.; Hajer, J.; Zavoral, M.; Lechuga, M.J.; Gerletti, P.; Tang, J.; Rosenstein, R.B.; Macdonald, K.; Bhadra, P.; Fowler, R.; Wittes, J.; Zauber, A.G.; Solomon, S.D.; Levin, B. Celecoxib for the prevention of colorectal adenomatous polyps. New England J. Med. 2006, 355, 855-995.

11. Harris, R.E. COX-2 blockade in the chemoprevention of cancers of the colon, breast prostate and lung. Inflammopharmacology 2009, 17, 55-67.

12. Terry, M.B.; Gammon, M.D.; Zhang, F.F.; et al. Association of frequency and duration of aspirin use and hormone receptor stauts with breast cancer risk. JAMA 2004, 20, 2433-2440.

13. Davies, G.; Martin, L.A.; Sacks, N.; Dowsett, M. Cyclooxygenase(COX-2), aromatase and breast cancer: a possible role for COX-2 inhibitors in breast cancer chemoprevention. Ann. Oncol. 2002, 13, 669-678. 
14. Harris, R.E.; Kasbari, S.; Farrar, W.B. Prospective study of non-steroidal anti-inflammatory drugs and breast cancer. Oncol. Rep. 1999, 6, 71-73.

15. Harris, R.E.; Chlebowski, R.T.; Jackson, R.D.; Frid, D.; Ascenseo, J.L.; Anderson, G.; Loar, A.; Rodabough, R.J.; White, E.; McTiernan, A. Breast cancer and non-steroidal anti-inflammatory drugs: prospective research from the women's health initiative. Cancer Res. 2003, 63, 6096-6101.

16. Marshall, S.F.; Bernstein, L.; Anton-Culver, H.; Deapon, D.; Horn-Ross, P.L.; Mohrenweiser, H.; Peel, D.; Pinder, R.; Purdie, D.M.; Reynolds, P.; Stram, D.; West, D.; Wright, W.E.; Ziogas, A.; Ross, R.K. Non-steroidal anti-inflammatory drug use and breast cancer risk by stage and hormone receptor status. J. Natl. Cancer Inst. 2005, 97, 805-812.

17. Harris, R.E.; Beebe-Donk, J.; Alshafie, G.A. Reduced risk of human lung cancer by selective cyclooxygenase 2 (COX-2) blockade: A case control study. Int. J. Biol. Sci. 2007, 13, 328-334.

18. Chen. J.H.; Liu, T.Y.; Wu, C.W.; Chi, C.W. Non steroidal anti-inflammatory drugs for the treatment of advanced gastric cancer: Cyclo-oxgenase 2 is involved in hepatocyte growth factor mediated tumour development and progression. Med. Hypotheses 2001.57, 503-505.

19. Ristimäki, A.; Sivula, A.; Lundin, J.; Lundin, M.; Salminen, T.; Haglund, C.; Joensuu, H.; Isola, J. Prognostic significance of elevated cyclooxygenase-2 expression in breast cancer. Cancer Res. 2002, 62, 632-635.

20. Subbaramaiah, K.; Norton, L.; Gerald, W.; Dannenberg, A.J. Cyclooxygenase is overexpressed in HER-2/neu-positive breast cancer: Evidence for involvement of AP-1 and PEA3. J. Biol. Chem. 2002, 277, 18649-18657.

21. Witton, C.J.; Hawe, S.J.; Cooke, T.G.; Bartlett, J.M. Cyclooxygenase 2 (COX2) expression is associated with poor outcome in estrogen receptor negative, but not estrogen receptor positive, breast cancer. Histopathology 2004, 45, 47-54.

22. Blair, C.K.; Sweeney, C.; Anderson, K.E.; Folsom, A.R. Nonsteroidal anti-inflammatory drug use and survival after breast cancer diagnosis in post-menopausal women. Breast Cancer Res. Treat. 2007, 101, 191-197.

23. Brodie, A.M.; Lu, Q.; Long, B.J.; Fulton, A.; Chen, T.; Macpherson, N.; DeJong, P.C.; Blankenstein, M.A.; Nortier, J.W.R.; Slee, P.H.T.J.; van de Ven, J.; van Gorp, J.M.H.H.; Elbers, J.R.J.; Schipper, M.E.I.; Blijham, G.H.; Thijssen, J.H. Aromatase and cyclooxygenase-2 expression in human breast cancers. J. Steroid Biochem. Mol. Biol. 2001, 79, 41-47.

24. Harris. R.E.; Alshafie, G.A.; Seibert, K. Chemoprevention of breast cancer in rats by celecoxib, a cyclooxygenase-2 inhibitor. Cancer Res. 2000, 60, 2101-2103.

25. Kubatka, P.; Ahlers, I.; Ahlersová, E.; Adámeková, E.; Luk, P.; Bojková, B.; Marková M. Chemopreventuon of mammary carcinogenesis in female rats by rofecoxib. Cancer Lett. 2003, 202, 131-136.

26. Mazhar, D.; Ang, R.; Waxman, J. COX inhibitors and breast cancer. Br. J. cancer 2006, 94, 346-350.

27. Zelnak. A.B.; O'Regan, R.M. Chemoprevention of breast cancer. Curr. Probl. Cancer 2004, 28, 201-217.

28. Singh-Ranger, G.; Mobkel, K. The role of cyclooxygenase-2 (COX-2) in breast cancer, and implications of COX-2 inhibition. Eur. J. Surg. Oncol. 2002, 28, 729-737. 
29. Muller-Decker, K.; Berger, I.; Ackermann, K.; Ehemann, V.; Zoubova, S.; Aulmann, S.; Pyerin, W.; Fürstenberger, G. Cystic duct dilatations and proliferative epithelial lesions in mouse mammary glands upon keratin 5 promoter-driven overexpression of cyclooxygenase-2. Am. J. Path. 2005, 166, 575-584.

30. Díaz-Cruz, E.S.; Brueggemeier, R.W. Interrealtionships between cyclooxygenases and aromatase: Unravelling the relevance of COX inhibitors in breast cancer. Anticancer Agents Med. Chem. 2006, 6, 221-232.

31. Zhao, Y.; Zhu, S.; Li, X.; Wang, F.; Hu, F.-L.; Li, D.-D.; Zhang, W.-C.; Li, X. Association between NSAID use and breast cancer risk: A systematic review and meta-analysis. Breast Cancer Res. Treat. 2009, 117, 141-150.

32. Khuder, S.A.; Mutgi, A.B. Breast cancer and NSAID se a meta-analysis. Br. J. Cancer 2001, 85, 1188-1192.

33. Mangiapne, S.; Blettner, M.; Schlattmann, P. Aspirin use and breast cancer risk: A meta-analysis and meta-regression of observational studies from 2001-2005. Pharmcoepidemiol. Drug saf. 17, 115-124.

34. Friis, S.; Thomassen, L.; Sørensen, H.T.; Tjønneland, A.; Overvad, K.; Cronin-Fenton, D.P.; Vogel, U.; McLaughlin, J. K.; Blot, W.J.; Olsen, J.H. Non-steroidal anti-inflammatory drug use and breast cancer risk: A Danish Cohort study. Eur. J. Cancer Prev. 2008, 17, 88-96.

35. Khalkhali-Ellis, Z.; Hendrix, M.J. Nitric oxide regulation of maspin expression in normal mammary epithelial and breast cancer cells. Am. J. Pathol. 1994, 263, 526-529.

36. Girish, G.V.; Sinha, N.; Chakraborty, K.; Bhattacharya, G.; Kahn, N.N.; Sinha, A.K. Restoration of impaired plasma maspin level in human breast cancer. Acta Oncol. 2006, 45, 526-529.

37. Kashfi, K.; Rigas, B. Molecular targets of nitric-oxide- donating aspirin in cancer. Biochem. Soc. Trans. 2005. 33, 701-704.

38. Cavalieri, E.L.; Rogan, E.G. Depurinating estrogen-DNA adducts in the etiology and prevention of breast ad other cancers. Future oncol. 2010, 6, 75-91.

39. Boland, G.P.; Butt, I.S.; Prasad, R.; Knox, W.F.; Bundred, N.J. Cox-2 expression is associated with an aggressive phenotype in ductal carcinoma in situ. Br. J. Cancer 2004. 90, 423-429.

40. Tan, K.B.; Yong, W.P.; Putti, T.C. COX-2 expression: A potential prognostic and predictive marker for high-grade ductal carcinoma in situ of the breast. Histopathology 2004, 44, 24-28.

41. Zhang, Y.Q.; Coogan, P.F.; Palmer, J.R.; Strom, B.L.; Rosenberg, L. Use of non-steroidal antiinflammatory drugs and risk of breast cancer: The case control surveillance study revisited. Am. J. Epidemiol. 2005, 162,165-170.

42. Kirsch, V.A.; Kreiger, N.; Cotterchio, M.; Sloan, M.; Theis, B. Non-steroidal anti-inflammatory drugs use and breast cancer risk: Subgroup findings. Am. J. Epidemiol. 2007, 166, 709-716.

43. McCarthy, K.; Bustin, S.A.; Ogunkolade, B.; Khalaf, S.; Laban, C.A.; McVittie, C.J.; Carpenter, R.; Jenkins, P.J. Cyclooxygenase-2 mRNA expression and hormone receptor staus in breast cancer. Eur. J. Surg. Oncol. 2006, 32, 707-709.

44. Sharp, C.R.; Collet, J.P.; McNutt, M.; Belzile, E.; Boivin, J.-F.; Hanley, J.A. Nested case-control study of the effects of nonsteroidal anti-inflammatory drugs on breast cancer risk and stage. $B$ r. $J$. Cancer 2000. 83, 112-120. 
45. Falandary, C.; Canney, P.A.; Freyer, G.; Dirix, L.Y. Role of combination therapy with aromatase and cox-2 inhibitors in patients with metastatic breast cancer. Ann. Oncol. 2009, 20, 615-620.

46. Daidone, M.G.; Coradini, D.; Martelli, G.; Veneroni, S. Primary breast cancer in elderly women: Biological profile and relayion with clinical outcome. Crit. Rev. Oncol. Haematol. 2003, 45, 313-325.

47. Gennari, R.; Curgliano, G.; Rotmensz, N.; Robertson, C.; Colleoni, M.; Zurrida, S.; Nolè, F.; de Braud, F.; Orlando, L.; Leonardi, M.C.; Galimberti, V.; Intra, M.; Veronesi, P.; Renne, G.; Cinieri, S.; Audisio, R.A.; Luini, A.; Orecchia, R.; Viale, G.; Goldhirsch, A. Breast carcinoma in elderly women. Cancer 2004, 101, 1302-1310.

48. Fisher, C.J.; Egan, M.K.; Smith, P.; Wicks, K.; Millis, R.R.; Fentiman, I.S. Histopathology of breast cancer in elation to age. Br. J. Cancer 1997, 75, 593-596.

49. Pike, M.C.; Krailo, M.D.; Henderson, B.E.; Casagrande, J.T.; Hoel, D.G. Hormonal risk factors, breast tissue age and the age-incidence of breast cancer. Nature 1983, 303, 767-770.

50. Johnson, T.W.; Anderson, K.E.; Lazovich, D.; Folsom, A.R. Association of aspirin and nonsteroidal anti-inflammatory drugs use drug use with breast cancer. Cancer Epidemiol. Biomarkers Prev. 2002, 11, 1586-1591.

51. Sauter, E.W.; Qin, W.; Schlatter, L.; Hewitt, J.E.; Flynn, J.T. Celecoxib decreases prostaglandin E 2 concentrations in the nipple aspirate fluid from high risk postmenopausal women and women with breast cancer. BMC Cancer 2006, 101, 191-197.

52. Agrawal, A.; Fentiman, I.S. NSAIDs and breast cancer: A possible prevention and treatment strategy. Int. J. Clin. Practice 2008, 62, 444-449.

(C) 2010 by the authors; licensee MDPI, Basel, Switzerland. This article is an Open Access article distributed under the terms and conditions of the Creative Commons Attribution license (http://creativecommons.org/licenses/by/3.0/). 